\title{
Prediction of Residual Pipeline Resource Taking into Account the Operation Loading Conditions
}

\author{
Yu. V. Banahevych, ${ }^{a}$ O. E. Andreykiv, ${ }^{b}$ and M. B. Kit ${ }^{\text {b }}$ \\ ${ }^{a}$ Department of Main Gas Pipelines "Lvivtransgas," Lviv, Ukraine \\ ${ }^{\mathrm{b}}$ Franko National University, Lviv, Ukraine
}

An effective energy approach to the evaluation of the residual service life of a pipe of oil pipeline containing a crack on its inner surface for the two-frequency loading mode of biaxial tensioncompression has been proposed. The two-frequency variations of pressure in the pipe are caused by the turbulence of the flow of oil (high frequency), opening and closing of the gate valves, and the shutdowns of the pumps (low frequency).

Keywords: residual service life of a pipeline, block loading, fatigue crack propagation, energy approach.

Introduction. Based on the energy approach developed in [1-4], we propose a method for the determination of the residual service life of structural elements with cracks under block loading by bilateral tension-compression. The proposed method is applied to the evaluation of the residual service life of a pipe with surface crack subjected to longitudinal bilateral tension-compression caused by the processes of heating and cooling of the pipe, its squeezing by the soil, and the action of internal pressure formed in the process of oil pumping. The two-frequency variations of pressure in the pipe are explained by the turbulence of the oil flow (high frequency), closing and opening of the gate valves, and shutdowns of the pumps (low frequency).

Model of Loading of a Pipe. The oil pressure in the main pipelines can be quite high to guarantee their high throughput capacity. The deviations of working pressure caused by the startups and shutdowns of the pump stations can be as large as 2-3 $\mathrm{MPa}$ [5]. In case of successive pumping of different types of oil, these deviations do not exceed $1 \mathrm{MPa}$, the deviations caused by the startups and shutdowns of separate aggregates vary within the range $0.5-1.0 \mathrm{MPa}$, and the deviations caused by the pollution of the pipeline and formation of air blocks, as a rule, do not exceed $0.5 \mathrm{MPa}$.

Due to the oil flow turbulence, a disbalance of the pumps, and the flow frequency oscillations, the pressure in the pipeline deviates from its mean value by $0.2-0.3 \mathrm{MPa}[6]$. The design-basis pressure for the reinforcement of pipelines at the intermediate pump stations is usually set equal to $4 \mathrm{MPa}$ because a pressure close to this level is formed after the shutdown of this station.

The restriction imposed on the input pressure of the station, i.e., $2.1 \mathrm{MPa}$, is connected with the action of a regulating valve of the protective system of the oil main. The rate of pressure increase depends on the response time of the pump rotor. Thus, the steepness of the shock wave decreases as the indicated response time increases. In the course of closing of a gate valve, the level of pressure specified by the flow interruption rate rapidly increases. The pressure wave C Yu. V. BANAHEVYCH, O. E. ANDREYKIV, M. B. KIT, 2009 
propagates with the sound speed toward the previous pump station, reflects from it, and, thus, leads to the formation of a wave of lower pressure. The amplitude of the pressure waves formed in the process of closing of gate valves can be as high as 4.44 $\mathrm{MPa}$ [5]. Thus, pressure variation in the pipes of oil mains is, in fact, two-frequency and can be simulated by block loading.

The experimental graphic dependence of pressure variations in a pipe in the process of repumping of oil [5] is qualitatively similar to the model diagram.

As a result of the increase in the diameters of oil mains, pressure, and temperature of oil products, the levels of stresses formed in the walls of the pipes become much higher, especially in the longitudinal direction. The changes in the properties of soil along the route of the pipeline result in different conditions of its squeezing. This may lead to the formation of significant longitudinal stresses $Q$ between fixed points of the pipeline. These stresses depend on temperature and pressure and can be found by using the following formula [7]:

$$
Q=\alpha E(\Delta T)-\frac{0.3 p d}{d_{2}-d_{1}},
$$

where $\alpha=12 \cdot 10^{-6} \mathrm{deg}^{-1}$ is the coefficient of linear thermal expansion of the pipe metal, $d_{2}=2 r_{2}$ and $d_{1}=2 r_{1}$, are, respectively, the inner and outer diameters of the pipe, $p$ is the pressure of oil products, $E$ is the elastic modulus, $\Delta T=T_{m}-T_{e}$, and $T_{m}$ and $T_{e}$ are the temperatures of the pipeline in the processes of installation and operation, respectively.

We consider a pipe containing a surface semielliptic crack (Fig. 1).

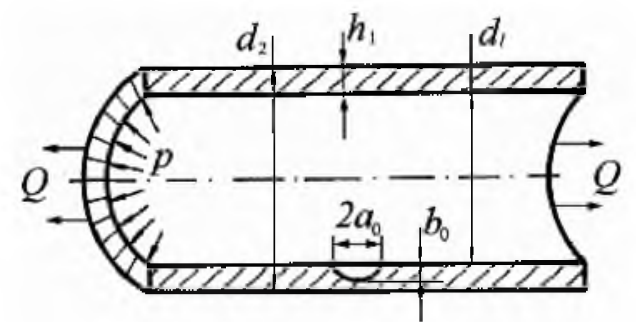

Fig. 1. Schematic diagram of loading of a cracked pipe.

According to the data of in-situ observations, the level of stresses $Q$ caused by the variations of temperature in the walls of the pipe restrained by soil can be as high as $200 \mathrm{MPa}$ [7]. Variations of pressure $p$ can be mathematically described as follows. The high-frequency oscillations of pressure $p$ are represented by the majorant

$$
p_{1}=a_{1}+b_{1} \sin \omega_{1} t
$$

where, according to the data of the in-situ observations [5], the measured quantities $a_{1}, b_{1}$, and $\omega_{1}$, have the following values: $a_{1}=1 \mathrm{MPa}, b_{1}=0.2 \mathrm{MPa}$, and $\omega_{1}=0.6 \mathrm{~Hz}$. The oscillations of pressure caused by the delays in the process of oil pumping due to the shutdowns of pumps, closing of gate valves, etc. are also described by a majorant sinusoidal cyclic curve of the form 


$$
p_{2}=a_{2}+b_{2} \sin \omega_{2} t
$$

where $a_{2}=3.5 \mathrm{MPa}, b_{2}=1 \mathrm{MPa}$, and $\omega_{2}=1.3 \mathrm{MHz}$ [6]. Hence, pressure variations in the pipeline $p(t)$ are described by the superposition of relations (1) and (2).

Since $\omega_{1}$ is much lower than $\omega_{2}$, the period of subcritical growth of the semielliptic crack is determined by the number of cycles $N_{2}=t / T_{2}$ of low-frequency oscillations.

Computational Model of Fatigue Crack Propagation. To determine the residual service life of the pipeline (the time to its depressurization), we propose computational models of the development of defects and determine the time of their penetration through the wall (Fig. 1).

Assume that the process of crack propagation is continuous. By analogy with [2], we write the equation of energy balance of the body at any time $t$ in the form $A=W+\Gamma$, where $A$ is the work of external forces, $W$ is the energy of deformation of the body, $W=W_{e}+W_{p}^{(1)}(S)+W_{p}^{(2)}(t)-W_{p}^{(3)}(t), W_{e}$ is the elastic component of $W, W_{p}^{(1)}(S)$ is the work of plastic strains depending only on the area of the crack $S, W_{p}^{(2)}(t)$ is the work of plastic strains caused by the external forces for a constant area of the crack during the incubation period of preparation to its jump depending only on time $t, W_{p}^{(3)}(t)$ is the work of plastic strains in the process of compression of the process zone (caused by the release of potential energy of the body also for a constant area of the crack during the incubation period of preparation to its next jump) depending only on time $t$, and $\Gamma$ is the fracture energy of the body depending solely on the area of the crack Since the requirement of energy balance is satisfied, the following condition of balance of the rates of variation of energy is also true:

$$
\begin{gathered}
\frac{\partial\left[\Gamma-\left(A-W_{e}-W_{p}^{(1)}-W_{p}^{(2)}+W_{p}^{(3)}\right)\right]}{\partial S} \frac{d S}{d t}- \\
-\frac{\partial\left(A-W_{e}-W_{p}^{(1)}-W_{p}^{(2)}+W_{p}^{(3)}\right)}{\partial t}=0 .
\end{gathered}
$$

Multiplying Eq. (3) by $T_{2}$ and differentiating the result with regard for the dependences of the functions on $S$ and $t$, we arrive at the following relation for the crack propagation rate $V=d S / d t$ :

$$
\frac{d S}{d N_{2}}=\frac{W_{C}^{(1)}}{\left(\gamma-\gamma_{s}\right)}
$$

where $N_{2}$ is the number of blocks of loading (low-frequency cycles), $\gamma$ is the density of fracture energy of the material $[2,3], \gamma_{s}$ is the density of potential energy in the process zone for the maximum level of stresses in a cycle, and 
$W_{C}^{(1)}$ is the cyclic component of the dissipation of energy in the process zone for one package of loading with $T_{2}=2 \pi \omega_{2}^{-1}$.

Since the thickness of the wall of the oil main $h_{1}=r_{2}-r_{1}$ is much smaller than the radius of the pipe $r_{1}$, for the sake of simplicity, we assume that the crack propagates in an infinite plate under the action of the static stresses $Q$ and variable loading $p$.

The quantities $\gamma, \gamma_{s}$, and $W_{C}^{(1)}$ are determined as follows [2, 3]:

$$
\begin{gathered}
\gamma_{s}=\frac{\varepsilon_{f c}}{\delta_{f c} \Delta S} \int_{L} d x \int_{0}^{l_{s}} \sigma_{0 f} \delta_{\max }(\xi, x) d \xi, \quad \gamma=0.75 \sigma_{0 f} \varepsilon_{f c}, \\
W_{C}^{(1)}=W_{C 1}^{(1)}+W_{C 2}^{(1)}, \\
W_{C 1}^{(1)}=\frac{\varepsilon_{f c}}{\delta_{f c}} \int_{0}^{N_{1} / 2} d y \int_{L} d x \int_{0}^{l_{f}} \sigma_{0 f}\left[\delta_{\max }(\xi, x, y)-\delta_{\min }(\xi, x, y)\right] d \xi, \\
W_{C 2}^{(1)}=\varepsilon_{f c} \delta_{f c}^{-1} \int_{0}^{l_{c}} \int_{L} \sigma_{0 f}\left[\delta_{\max }(\xi, x, 0)-\delta_{\operatorname{Imin}}(\xi, x, 0)\right] d x d \xi,
\end{gathered}
$$

where $\sigma_{0 f}$ is the mean level of stresses in the process zone under the loads $p$ and $Q, \Delta S$ is the area of the process zone near the crack contour, $y$ is the number of cycles of oscillations of pressure $p(t)$ for the period $T_{2}, \delta_{\max }$ and $\delta_{\min }$ are, respectively, the maximum and minimum values of the opening displacement of the model cut along the process zone [2], $\delta_{f_{c}}$ is its critical value for the critical strain $\varepsilon_{f_{c}}$ under cyclic loading, $d \xi$ is an increment of arc length along the crack contour $L, l_{f}$ and $l_{s}$ are the widths of the cyclic and static process zones, respectively, and $l_{c}$ is the critical value of $l_{s}$.

By using the Huber-Mises condition of plasticity, we determine the quantity $\sigma_{0 f}$ as follows [3]:

$$
\sigma_{0 f}=0.5\left[Q+\sqrt{\left(\sigma_{s}+\sigma_{p}\right)^{2}-3 Q^{2}}\right],
$$

where $\sigma_{s}$ and $\sigma_{p}$ are, respectively, the ultimate and yield strengths of the material.

To simplify the solution of the problem, we assume that the quantity $\delta_{\max }-\delta_{\min }$ is identical in the course of increase and decrease in the load, which is used to compute the number of cycles to failure. Thus, by using the results obtained in $[2,3]$, we find

$$
\begin{gathered}
\delta_{\max }-\delta_{\min }=0.5 \delta_{\max }(1-R)^{2}=\frac{0.5 K_{I \max }^{2}(1-R)^{2}}{E \delta_{0 f}}, \\
l_{f}-0.25 l_{s}(1-R)^{2}=\frac{0.1 K_{I \max }^{2}(1-R)^{2}}{\sigma_{0 f}^{2}},
\end{gathered}
$$


where $R=p_{\min } / p_{\max }$ is the load ratio. Note that $R$ is a function of $N_{1}$. These relations and the results presented in $[2,3]$ enable us to rewrite relations $(5)$ in the form

$$
\begin{gathered}
W_{C 2}^{(1)}=\frac{0.0368 \alpha \beta \varepsilon_{f c}}{K_{f c}^{2} \sigma_{0 f}} \int_{L}\left[K_{I \max }^{(1)}(\xi, 0)-K_{I \min }(\xi, 0)\right]^{4} d \xi \\
\gamma_{s}=\frac{0.2944 \varepsilon_{f c}}{K_{f c}^{2}(\Delta S) \sigma_{0 f}^{1}} \int_{L}\left(K_{[\max }^{(2)}(\xi, 0)\right)^{4} d \xi \\
\Delta S=\frac{0.3927}{\sigma_{0 f}^{2}} \int_{L}\left(K_{\mathrm{Imax}}^{(2)}(\xi, 0)\right)^{2} d \xi \\
W_{C 1}^{(1)}=0.0368 \alpha \beta \varepsilon_{f c} K_{f c}^{-2} \sigma_{0 f}^{-1} \int_{L} d \xi \int_{0}^{N_{1} / 2} 2\left[K_{\operatorname{Imax}}(\xi, y)-K_{\mathrm{I} \min }(\xi, y)\right]^{4} d y .
\end{gathered}
$$

where $K_{\operatorname{Imax}}(\xi, 0)$ and $K_{I \min }(\xi, 0)$ are the maximum and minimum values of $K_{\mathrm{I}}$ for cycles of the second type and $K_{\mathrm{Imax}}(\xi, y)$ and $K_{\mathrm{Imin}}(\xi, y)$ are the maximum and minimum values of $K_{\mathrm{I}}$ for cycles of the first type.

We apply the method of equivalent areas $[2,3,8]$, according to which the increment of the area of a fatigue crack of a given configuration is approximately equal to the increment of the area of a circular crack of radius $a$ (of the same area). This enables us to write the approximate values of $K_{\operatorname{Imax}}(\xi, 0), K_{\operatorname{Imin}}(\xi, 0)$, $K_{\text {Imax }}(\xi, y)$, and $K_{I \min }(\xi, y)$ by analogy with the case of a plate with surface semicircular crack stretched by the uniformly distributed stresses $\sigma=r_{1} h_{1}^{-1} p(t)$ as follows:

$$
\begin{gathered}
K_{\mathrm{Imax}}^{(i)}(\xi, 0)=2 r_{1} h_{1}^{-1} \pi^{-1 / 2} a^{1 / 2}\left(a_{2}+b_{2}+(-1)^{i} b_{1}\right) \quad(i=1,2), \\
K_{\mathrm{I} \max }(\xi, 0)=2 \pi^{-1 / 2} r_{1} h_{1}^{-1} a^{1 / 2}\left(a_{2}-b_{2}-b_{1}\right), \\
K_{\mathrm{I} \max }(\xi, y)=2 \pi^{-1 / 2} r_{1} h_{1}^{-1} a^{1 / 2}\left[a_{2}+b_{1}+b_{2} \sin \left(2 \pi y / N_{1}-\pi / 2\right)\right], \\
K_{\mathrm{I} \max }(\xi, y)=2 \pi^{-1 / 2} r_{1} h_{1}^{-1} a^{1 / 2}\left[a_{2}-b_{1}+b_{2} \sin \left(2 \pi y / N_{1}-\pi / 2\right)\right] .
\end{gathered}
$$

Further, we substitute relations (9) in (8) and then, together with (5), in (4). This yields

$$
\frac{d a}{d N_{2}}=\frac{1.3 \alpha \beta a^{2} r_{1}^{4} h_{1}^{-4}\left[b_{2}^{4}+b_{1}^{4} N_{1}\right]}{\sigma_{0 f}^{2}\left[K_{f_{c}}^{2}-1.2732 a r_{1}^{2} h^{-2}\left(a_{2}+b_{2}+b_{1}\right)^{2}\right]} .
$$

Thus, we have deduced the differential equation (10) for the evaluation of subcritical crack growth in the case where the pipe is depressurized. This equation describes only the kinetics of fatigue crack propagation. To complete the mathematical model, we supplement it with the following initial and final conditions $[2,3]$ : 


$$
\begin{gathered}
N_{2}=0, \quad a=\sqrt{\pi^{-1} S_{0}}, \\
N_{2}=N_{g}, \quad a=h_{1},
\end{gathered}
$$

where $S_{0}$ is the area of the initial crack. For small surface elliptic cracks [3], the ratio of the semiaxes $a_{0}$ and $b_{0}$ of the ellipse takes the following approximate value: $x=b_{0} / a_{0} \approx 0.7$ and, hence, $N_{2}=0$ and $a=1.2 a_{0}$, where $2 a_{0}$ is the length of the crack on the surface of the pipe wall.

Thus, the mathematical model used to predict the operation period prior to the depressurization of a pipe of oil main includes relations (10)-(12). The material constants $\alpha, \beta, K_{f c}$, and $\sigma_{0 f}$ are determined experimentally [2, 3].

Pipe Residual Life Assessment. Integrating the differential equation of growth of a fatigue crack (10) with initial (11) and final (12) conditions, we get

$$
N_{g}=\frac{C_{2}}{C_{1}}\left(\frac{1}{a_{0}}-\frac{1}{h_{1}}\right)+\frac{C_{3}}{C_{1}} \ln \left(\frac{a_{0}}{h_{1}}\right),
$$

where

$$
\begin{gathered}
C_{1}=1.2726 \alpha \beta r_{1}^{4} h_{1}^{-4}\left(b_{2}^{4}+b_{1}^{4} N_{1}\right), \quad C_{2}=\sigma_{0 f}^{2} K_{f c}^{2}, \\
C_{3}=1.2732 \sigma_{0 f}^{2} r_{1}^{2} h_{1}^{-2}\left(a_{2}+b_{2}+b_{1}\right)^{2} .
\end{gathered}
$$

We now substitute the data obtained for the pipe of the KremenchugKherson oil main [3], i.e., $a_{1}=0, a_{2}=3.5 \mathrm{MPa}, b_{1}=0.15 \mathrm{MPa}, b_{2}=1 \mathrm{MPa}$, $r_{1}=0.5 \mathrm{~m}, h_{1}=0.012 \mathrm{~m}, Q=200 \mathrm{MPa}, \sigma_{Y}=420 \mathrm{MPa}, \sigma_{s}=880 \mathrm{MPa}, N_{1}=$ $=28 \cdot 10^{4}$ cycles, $K_{f c}=86 \mathrm{MPa} \sqrt{\mathrm{m}}$, and $\alpha \beta=3 \cdot 10^{-4}$ cycle $^{-1}$, in relation (13). This yields

$$
N_{g}=7.13\left(a_{0}^{-1}-83.33\right)+46.07 \ln \left(83.33 a_{0}\right) .
$$

In the absence of longitudinal tension-compression of the pipe, i.e., for $a=0$, we find

$$
N_{g}=5.7\left(a_{0}^{-1}-54.82\right)+36.88 \ln \left(a_{0}\right) \text {. }
$$

Further, in the absence of both longitudinal tension-compression of the pipe $(a=0)$ and hydraulic oscillations of pressure $\left(b_{1}=0, N_{1}=0\right)$, we obtain

$$
N_{g}=814.66\left(a_{0}^{-1}-56.57\right)+4930.36 \ln \left(a_{0}\right) .
$$

Relations (14)-(16) are used to construct the dependences of the residual service life $N_{g}$ on the size of the initial defect $a_{0}$ on the inner surface of the oil pipeline (Fig. 2).

In the case where the longitudinal tension-compression of the pipe and hydraulic oscillations of the pressure of oil are neglected, the residual service life of the pipe specified by relation (16) is too long to be regarded as realistic. 


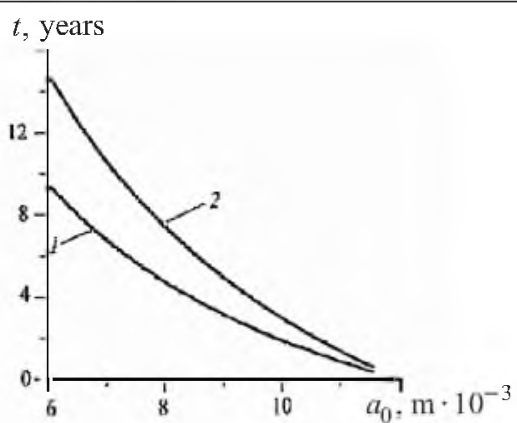

Fig. 2. Dependences of the residual life of the pipe on the initial size of the defect $a_{0}$ computed according to relations (14) (curve l) and (15) (curve 2).

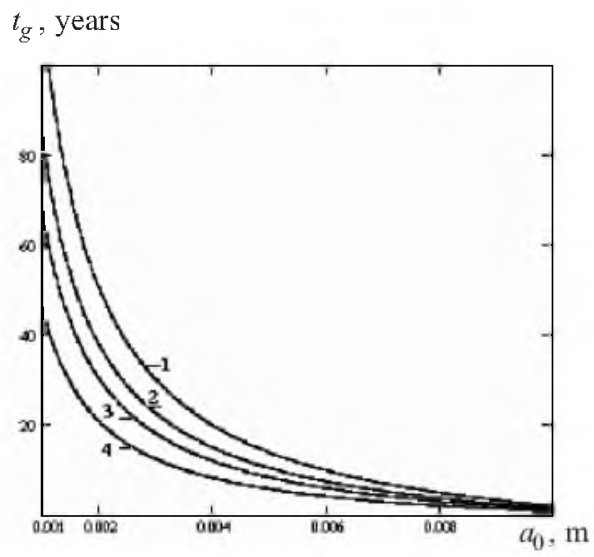

Fig. 3

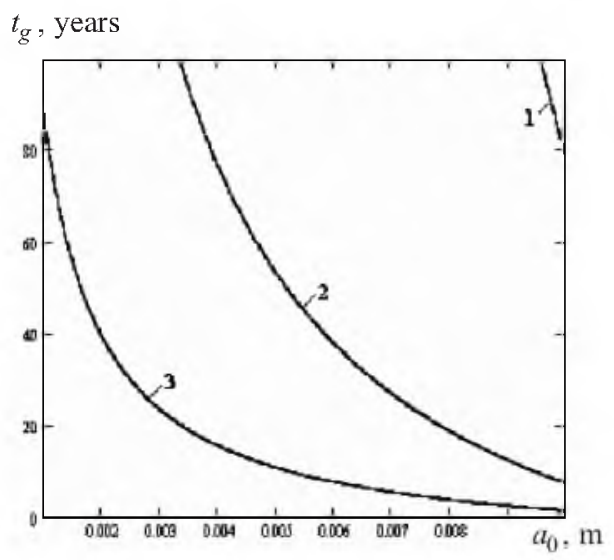

Fig. 4

Fig. 3. Dependence of residual lifetime of pipe $t_{g}$ on the defect initial size $a_{0}$ at the change of frequency $n$ of bolts opening-closing [(1) $n=30 ;$ (2) $n=40$; (3) $n=50$; (4) $n=73$.

Fig. 4. Dependence of residual lifetime $t_{g}$ of pipe on the defect initial size $a_{0}$ at the change of pressure $p$ amplitude $b_{1}$ on flow turbulence $\left[(1) b_{1}=0.05 ;(2) b_{1}=0.1 ;(3) b_{1}=0.15\right]$.

Optimum Operation Parameters for Oil Repumping. For the choice of optimum operating parameters of repumping oil, which could provide reliable lifetime of pipe of oil pipeline with set imperfectness, we study the effect of each of such parameters on the period $t_{g}$ of subcritical crack propagation in a pipe. For this purpose, we'll take advantage of dependences (13) and for each of such parameters we will write down these dependences in such kind:

change of frequency $n$ in the pipe of oil pipeline of closing and opening of bolts per year

$$
t_{g}=\frac{N_{g}}{n}, \quad \text { where } \quad n=30,40,50,73,
$$

change of amplitude $b_{1}$ of pressure in a pipe at turbulence of oil flow

$$
t_{g}=\frac{N_{g}\left(b_{1}\right)}{n}, \quad \text { where } \quad b_{1}=0.05,0.1,0.15 \text {, }
$$




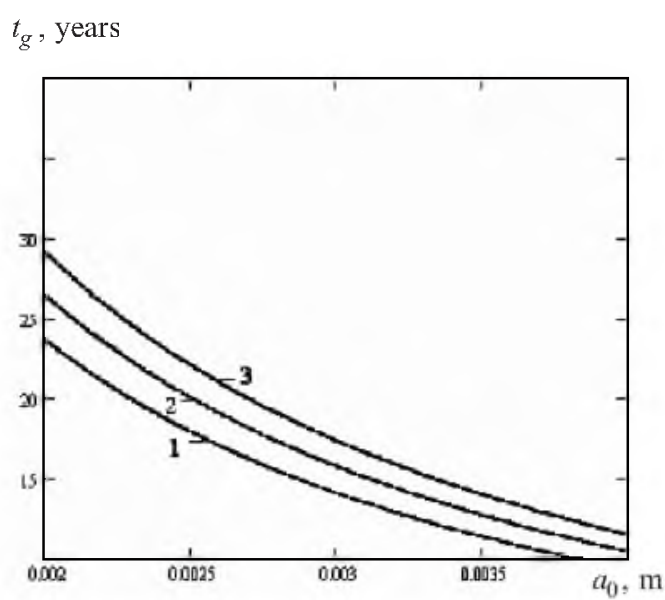

Fig. 5. Dependence of residual lifetime $t_{g}$ of pipe on the defect initial size $a_{0}$ at the change of size of tensions $Q$ in a pipe under its squeezing by the soil [( $I$ ) $Q=-150 \mathrm{MPa}$; (2) $Q=-100 \mathrm{MPa}$; (3) $Q=-50 \mathrm{MPa}$.

change of size of tensions $\sigma$ in the wall of pipe from its squeezing by the soil

$$
t_{g}=\frac{N_{g}(Q)}{n}, \quad \text { where } \quad Q=-150,-100,-50 \text {. }
$$

Graphic dependences of the residual lifetime of pipe of oil pipeline on the above parameters are presented in Figs. 3-5 using formulas (17)-(19).

Conclusions. From the analysis of graphic dependences (see Fig. 3-5) it is possible to draw the following conclusions:

(i) the decrease of a number of the bolts closing-opening increases the pipe lifetime;

(ii) presence of turbulence of the oil flow in the pipe decreases its lifetime, which requires best synchronization of pumps' operation;

(iii) the decrease of pipe's squeezing by the soil results in the increase of the pipe lifetime.

1. J. Schijve, "Fatigue of materials and structures in the 20th century: State-ofthe-art," Mater. Sci., 39, No. 3, 7-27 (2003).

2. M. Shata and Z. Terlets'ka, "Energy approach in the mechanics of fatigue propagation of macrocracks," in: V. V. Panasyuk (Ed.), Fracture Mechanics of Materials and Strength of Structures [in Ukrainian], Issue 2, Kamenyar, Lviv (1999), pp. 141-148.

3. Z. O. Terlets'ka, Evaluation of the Service Lufe of Structural Elements with Surface Cracks under the Action of Variable Loads and Corrosive Media [in Ukrainian], Author's Abstract of the Candidate Degree Thesis (Tech. Sci.), Ternopil (2002).

4. O. E. Andreykiv and M. V. Lishchyns'ka, "Equation of growth of fatigue cracks in inhomogeneous plates," Mater. Sci., 35, No. 3, 53-58 (1999). 
5. V. M. Agapkin and B. L. Krivoshein, Methods for the Protection of Pipelines Against Failures in Nonsteady Modes [in Russian], VNIIOENG, Moscow (1976).

6. L. V. Zaitsev, Regulation of the Modes of Operation of Oil Mains [in Russian], Nedra, Moscow (1982).

7. L. G. Karpova and K. E. Rashchepkin, "Investigation of the elastoplastic interaction of pipelines with soil," in: Pipeline Transport of Oil and Oil Products [in Russian], VNIISPNeft', Ufa (1974), pp. 120-128.

8. A. E. Andreykiv and A. I. Darchuk, "A method for the integral evaluation of the durability of a structural element containing a crack propagating in a plane," Mater. Sci., 24, No. 6, 53-56 (1988).

Received 11. 06. 2008 